\title{
Delirium:
}

\section{la confusión de los clínicos}

\author{
Matías G onzález $T^{1,2, a}$, Joan de Pablo $R^{1}$, \\ Manuel Valdés $\mathbf{M}^{3}$. \\ The clinical confusion about \\ delirium
}

Delinium or acute confusional state is a condition that lies within the boundaries of psychiatry and other medical specialties. It is defined as a syndrome characterized by a fluctuating cognitive impairment of acute onset. The pathogenesis is multifactorial and it frequently appears in elderly patients admitted to general hospitals. Delirium carries a high mortality and it prolongs hospital stay. Its diagnosis if often overlooked and the treatment is inadequate or belated. This article discusses the most efficient procedures to diagnose and treat delirium. The review was based on a systematic search in the literature using the key words delirium, acute mental syndrome, acute confusional state and organic mental syndrome. Articles were selected according to their relevance and methodological accuracy (Rev Méd Chile 2003; 131: 1051-60).

(Key Words: Brain diseases; Delirium, dementia, amnestic, cognitive disorders; Frail elderly).

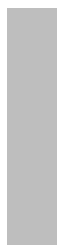

Recibido el 11 de marzo, 2003. Aceptado en versión corregida el 10 de julio, 2003.

${ }^{1}$ Sección de Psiquiatría de Enlace del Instituto Clínic de Psiquiatría y Psicología, Hospital Clinic Universitari de Barcelona. ${ }^{2}$ Departamento de Psiquiatría, Hospital Clínico Pontificia Universidad Católica de Chile. ${ }^{3}$ Instituto Clínico de Psiquiatría y Psicología, Hospital Clínic Universitari de Barcelona.

aDoctorado, Programa de Neurociencias de la Universidad de Barcelona. Fellowship de Psiquiatría de Enlace. Becario, Ministerio Asuntos Exteriores.

$E^{1}$ delirium es un trastorno que ha sido descrito desde el inicio de la literatura médica ${ }^{1}$ como un cuadro clínico, de inicio brusco y curso fluctuante, que se caracteriza por alteraciones de la conciencia, la atención y el pensamiento, y en el que pueden aparecer ideas delirantes y alucinaciones. Es un trastorno que se sitúa en la frontera entre la psiquiatría y el resto de especialidades médicas, puesto que puede aparecer en el curso

Correspondencia a: Dr. Joan de Pablo R. Av. Villarroel 170, Barcelona. CP: 08036. E mail: JDEPABLO@clinic.ub.es de muy diversas patologías. Se presenta con mucha frecuencia en los pacientes hospitalizados, sobre todo, de edad avanzada, y supone una importante complicación que se asocia a una elevada mortalidad y a un importante consumo de recursos, con elevada estancia media ${ }^{2,3}$.

Una de las peculiaridades de este síndrome ha sido la gran diversidad de términos con que se ha denominado (reacción exógena aguda, estado oniroide, síndrome confusional agudo, síndrome mental orgánico, síndrome mental agudo) lo cual, unido a una clínica caracterizada por una variada alteración de las funciones superiores, ha contribuido a generar una considerable confusión entre 
los clínicos. Afortunadamente, en el curso de la última década, se ha ido generando un consenso internacional para considerar que el término delirium es el más adecuado. Así, utilizan esta denominación las más importantes clasificaciones nosológicas ${ }^{4,5}$, los más importantes grupos de investigación especializados $\mathrm{s}^{6-9}$ y la base de datos Medline, en la que se constató este término en $91 \%$ de los trabajos publicados sobre el tema en los últimos 10 años. En consecuencia, puede decirse que el término delirium es actualmente el más adecuado para describir los estados confusionales agudos asociados a trastornos orgánicos cerebrales ${ }^{10}$.

\section{EPIDEMIOLOGÍA, FACTORES DE RIESGO Y COSTES}

La prevalencia del delirium en los pacientes hospitalizados aumenta con la edad y varía según el diagnóstico de ingreso (Tabla 1); también es el segundo síndrome psiquiátrico más prevalente en el ámbito hospitalario (10-30\% de los pacientes ingresados) ${ }^{11}$, después de los trastornos depresivos. Se ha señalado un aumento progresivo de su aparición en la población mayor de 65 años, con cifras que oscilan entre $10 \%$ y $56 \%$, asociándose a una mortalidad con rangos entre $10 \%$ y $65 \% 6-12$. Según un trabajo reciente efectuado en un hospital general de nuestro medio (España), la preva- lencia/día de delirium sería de $11,9 \%$, con una mortalidad de $23 \%$ durante el ingreso ${ }^{13}$. Por otro lado, en el contexto de una investigación multinacional europea realizada con 3.608 pacientes en seis centros hospitalarios españoles se detectó una prevalencia de $9,1 \%{ }^{14}$. Sin embargo, hay datos de que se trata de una emergencia médica que no se identifica adecuadamente en $32-67 \%$ de los casos y que acostumbra a ser objeto de un abordaje terapéutico tardío ${ }^{15}$.

Algunas patologías tienen más probabilidades de asociarse a delirium que otras y existen grupos de pacientes con un riesgo mayor. En un estudio realizado por Fann et $\mathrm{al}^{16}$, se investigó la prevalencia e incidencia de delirium en 90 pacientes en fase aguda de trasplante hematopoiético de stem cell, y se concluyó que $50 \%$ de los pacientes presentaban un episodio de delirium en las cuatro semanas siguientes al trasplante. González y Barros $^{17}$ estudiaron prospectivamente a 64 pacientes de todas las edades, en una unidad de cuidados intermedios y detectaron una prevalencia de delirium de $41 \%$. En un seguimiento realizado por Litaker et al $^{18}$, a 500 pacientes que iban a ser sometidos a una intervención quirúrgica electiva, se detectó delirium en $11,5 \%$ de los casos durante los cuatro días posteriores a su intervención. En otro estudio prospectivo, ciego y randomizado ${ }^{19}$, se estudió la prevalencia de delirium en pacientes mayores de 65 años admitidos de urgencia por

Tabla 1. Prevalencia de delirium en pacientes hospitalizados por diferentes causas

\begin{tabular}{|c|c|c|c|c|}
\hline Estudio & $\mathrm{n}^{0}$ paciente & Tipo de problema & Prevalencia & Incidencia \\
\hline \multirow[t]{2}{*}{ Inouye (1993) } & 107 & Médico, edad >70 & $\cdots$ & $25 \%$ \\
\hline & & Médico, edad >70 & $\cdots$ & $17 \%$ \\
\hline \multirow[t]{2}{*}{ Levkoff (1992) } & 325 & Urgencias edad $>70$ & $10,5 \%$ & $\ldots$ \\
\hline & 291 & Urgencias edad $>70$ & $\ldots$ & $31,3 \%$ \\
\hline Fann (2002) & 90 & $\begin{array}{l}\text { Fase aguda de trasplante } \\
\text { hematopoiético de stem cell } \\
\text { edad }>18\end{array}$ & $\cdots$ & $50 \%$ \\
\hline González (2000) & 64 & $\begin{array}{l}\text { Unidad cuidados intermedios } \\
\text { edad }>18\end{array}$ & $41 \%$ & $\cdots$ \\
\hline Marcantonio (2001) & 126 & $\begin{array}{l}\text { Fractura de fémur edad }>65 \text { : } \\
\text { - con protocolo prevención delirium } \\
\text { - sin protocolo prevención delirium }\end{array}$ & $\begin{array}{l}32 \% \\
50 \%\end{array}$ & - . \\
\hline
\end{tabular}


fractura de fémur, separados en dos grupos aleatorios. Uno que fue objeto de una intervención protocolizada que consistía en visitas y recomendaciones diarias de un geriatra, y el otro que sirvió de control. En el primer grupo la prevalencia de delirium fue de $32 \%$ y en el segundo grupo (sin intervención) fue de $50 \%$. Por otro lado, Uldall y Berghuis ${ }^{20}$ estudiaron retrospectivamente la frecuencia de delirium en 137 pacientes con SIDA, detectando al menos un episodio de delirium en $46 \%$ de los casos.

Por tanto, tendrán mayor riesgo de presentar delirium los pacientes sometidos a intervenciones quirúrgicas mayores (ej: tras intervención quirúrgica en fémur y cadera, cirugía cardiovascular, trasplante de órganos sólidos), los que sufren procesos infecciosos generalizados (ej: VIH, sepsis), los pacientes con cualquier tipo de alteración o lesión en el sistema nervioso central (ej: accidente vascular cerebral (AVC), traumatismo craneal, enfermedad de Parkinson o toxicomanías), pacientes oncológicos, pacientes terminales, pacientes polimedicados y pacientes con alteraciones hidroelectrolíticas.

Así pues, a partir de los estudios existentes, se puede concluir que el grupo de pacientes con mayor riesgo de presentar delirium sería el constituido por pacientes mayores de 65 años, en general con algún grado de deterioro neuropsicológico previo y con patología médica añadida, que están expuestos a diversos factores de riesgos intrínsecos al proceso mórbido y a su tratamiento. Además, cuantos más factores de riesgo confluyan (Tabla 2), más probable será la aparición del delirium ${ }^{9}$ 12,21,22. El abordaje y control de los factores de riesgo de delirium han sido objeto de investigación reciente: Inouye y Charpentier ${ }^{12}$ realizaron un estudio con una cohorte de 196 pacientes, con el fin de identificar los potenciales factores precipitantes de delirium en pacientes hospitalizados de más de 70 años. Lograron aislar cinco factores de riesgo para delirium (contención física, malnutrición, uso de más de tres fármacos, catéter vesical y evento iatrogénico), con los que establecieron niveles de riesgo de presentar delirium. Estos factores precipitantes y la vulnerabilidad basal fueron correlacionados con la presencia de delirium.

Por lo demás, el delirium incrementa las complicaciones médicas (infecciones intrahopitalarias, aspiraciones bronquiales, úlceras de decú- bito, etc.) y en ocasiones, puede establecerse un daño cerebral permanente con deterioro cognoscitivo irreversible. En el estudio de McCusker et $a^{23}$ se demostró que, tras el ajuste del resto de las variables, el padecimiento de delirium fue el mejor predictor independiente de deterioro cognoscitivo y de mal funcionamiento general a los dos años de la hospitalización. En otro estudio se investigó si el delirium contribuía a una peor evolución tras la hospitalización en pacientes mayores de 65 años, y para ello se estudió una cohorte de 725 pacientes, $12 \%$ de los cuales había presentado delirium a su ingreso. Una vez más se constató que el delirium era el mejor factor predictor de muerte, mal pronóstico, mayores demandas de enfermería tras el alta y declive en el funcionamiento general ${ }^{24}$.

En la actualidad, el modelo con más poder para integrar los distintos factores de riesgo de delirium se apoya en el concepto de "reserva cerebral"9 como capacidad del sistema nervioso central para responder de manera funcionalmente flexible a las agresiones. $\mathrm{Si}$ el sujeto ya parte basalmente de una reserva cerebral exigua como

\section{Tabla 2. Factores de riesgo para delirium}

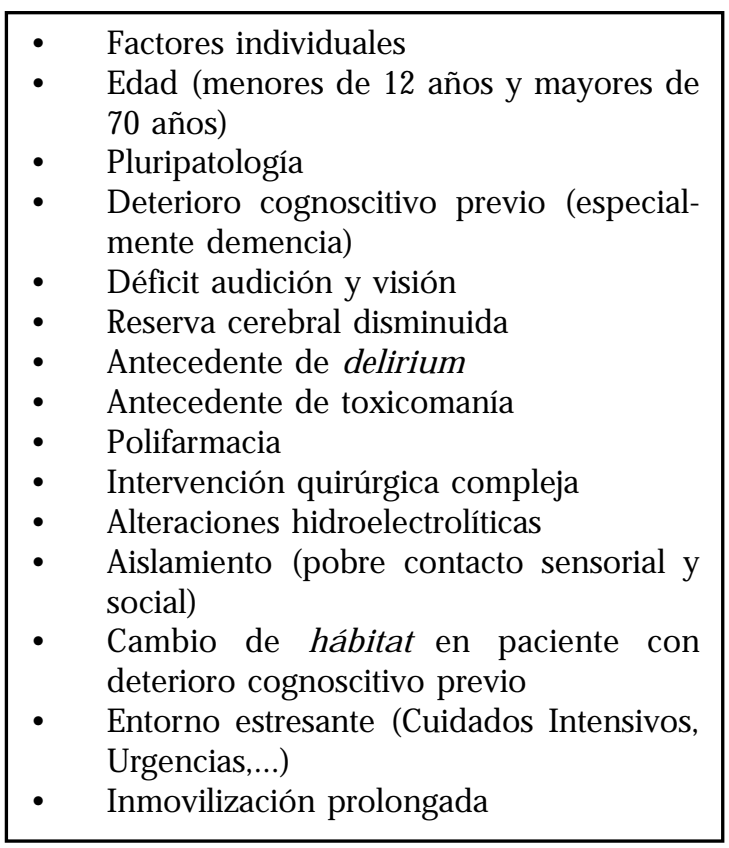


consecuencia de circunstancias previas, puede presentar delirium con mayor facilidad ante la presencia de factores de riesgo, y asimismo sus secuelas podrán ser mayores.

En cuanto a los costes sanitarios del delirium, cabe mencionar el incremento significativo de la ocupación hospitalaria (17,5 días de promedio de estancia versus 12 días en pacientes médicos con otros diagnósticos psiquiátricos) ${ }^{13}$, y un costo muy elevado, que en Estados Unidos de Norteamérica se estima puede llegar a 17,5 millones de dólares diarios, más el costo adicional de incremento de cuidados paliativos tras el alta ${ }^{25}$. Los autores de este último estudio señalaron que estos gastos podrían recortarse en $40 \%$ con medidas de prevención del delirium, con un diagnóstico más precoz y con terapéuticas más adecuadas.

\section{Eтiología}

El delirium raramente es causado por un único factor etiológico (Tabla 3), sino que suele ser resultado de la concurrencia de una reserva cere- bral disminuida que actúa como factor de predisposición y factores que desencadenan y mantienen el estado de disfunción cerebral $^{8}$. Los factores desencadenantes más frecuentes se relacionan con un desequilibrio metabólico, abstinencia a sustancias de abuso, fallo funcional de órganos vitales (insuficiencia hepática, renal, respiratoria 0 cardiocirculatoria), reacciones adversas a fármacos o bien una combinación de varios de estos factores.

En la última clasificación de la Asociación Americana de Psiquiatría ${ }^{4}$ se diferencian cuatro subtipos de delirium según su etiología: a) debido a una enfermedad médica; b) inducido por sustancias; c) debido a múltiples etiologías, y d) no especificado, si no se encuentra etiología de base. En cualquier caso, el estudio de los posibles factores causales del delirium determinará la naturaleza de la intervención terapéutica, que ha de tener en cuenta los diversos hallazgos clínicobiológicos.

Diversos trabajos desarrollados en los últimos años permiten efectuar algunas consideraciones sobre la fisiopatología del delirium que se resumen a continuación.

\section{Tabla 3. Causas más frecuentes de delirium}

- Alteraciones metabólicas: acidosis, alcalosis, alteración hidroelectrolítica, hipoxia de cualquier origen (anemias, fallo cardiopulmonar).

Infecciones: encefalitis, meningitis, sífilis, VIH.

- Paciente de urgencia: politraumatismo, infarto agudo al miocardio, quemadura severa, postoperatorio.

- Síndrome de privación o intoxicación: alcohol (delirium tremens), benzodiacepinas, barbitúricos, opiáceos, medicamentos, pesticidas, metales pesados.

Patología del SNC: tumores, hemorragias, hematoma subdural, hidrocefalia, TEC, abscesos.

Déficit vitamínicos: tiamina, ácido fólico, vitamina B12, niacina.

Cardiopulmonar: encefalopatía hipertensiva, infarto agudo miocardio, insuficiencia respiratoria aguda.

- Endocrinopatías: hiper o hipo glicemia, hiper o hipo adrenocorticoidismo, hiper o hipo tiroidismo.

- Fallo sistema renal: insuficiencia renal aguda o crónica, paciente en espera de diálisis 0 trasplante.

Fallo hepático: encefalopatía hepática, cirrosis, hepatitis.

Cirugía: trasplante, cirugía de cadera, cardiotomía.

- Cirugia: traspión. tranquilizantes, quimioterápicos,

- $\quad$ Polimedicación: tranquilizantes, quimioterápicos, anticolinérgicos, narcóticos y corticoides. 
Neuroanatomía. La diversidad de las manifestaciones clínicas del delirium sugiere que muchas áreas del cerebro están implicadas en su génesis ${ }^{10}$. Los estudios con neuroimagen muestran alteraciones como atrofia o accidentes cerebrovasculares a nivel tanto córtico como subcortical ${ }^{26}$, implicando especialmente áreas de la corteza frontal, tálamo anteromedial, ganglios basales derechos, corteza temporo-occipital basal-medial y corteza parietal posterior derecha ${ }^{7}$. Los estudios con PET y SPECT han comunicado resultados contradictorios en cuanto a las zonas cerebrales con aumento 0 disminución de flujo sanguíneo cerebral ${ }^{26}$, pero parecen apoyar la hipótesis de una disfunción del hemisferio derecho. En este campo de estudio cabe esperar un mayor progreso con el diseño de las investigaciones más recientes, que combinan las exploraciones de neuropsicología con las de neuroimagen.

Neurotransmisión. Aún es poco lo que se sabe de las alteraciones de la neurotransmisión en los estados de delirium $\mathrm{y}$, dada la diversidad de alteraciones estructurales y funcionales que lo acompañan, es muy poco probable que se trate de la alteración específica de un neurotransmisor determinado. Hasta ahora la neurotransmisión colinérgica ha recibido especial atención por estar particularmente implicada en procesos de memoria y atención. La hipótesis de la disregulación en la vía colinérgica se basa en el hecho de que el delirium se presenta en respuesta a hipofunciones colinérgicas de cualquier causa y en que las alteraciones cognoscitivas covarían con el nivel de anticolinérgicos en plasma ${ }^{26,27}$. Además, los fármacos anticolinérgicos producen síntomas mediados por el bloqueo de receptores postsinápticos, inhibición de liberación presináptica 0 acciones de metabolitos muscarínicos ${ }^{28}$. También se ha demostrado que los pacientes hospitalizados con delirium presentan mayores niveles de bloqueo colinérgico que los pacientes sin delirium ${ }^{29}$ y que la pérdida de plasticidad muscarínica y colinérgica aumenta con la edad ${ }^{30}$.

Por otra parte, hay estudios que sugieren que en los estados de delirium habría también una hipofunción dopaminérgica asociada a la colinérgica, en vista de la buena respuesta del delirium a los fármacos neurolépticos, que bloquean los receptores dopaminérgicos de manera relativa- mente específica. Además, en modelos animales ha podido demostrarse que la hipoxia neuronal se asocia a disminución de la liberación de acetilcolina y a incremento en la liberación de dopamina ${ }^{26}$.

En cuanto a la serotonina, cabe suponer su participación en biología del delirium, dado su papel relevante en la biología del sueño, en la regulación de la conducta motora, en la memoria y en la generación de estados alucinatorios (como los inducidos por el LSD). Lo mismo cabe decir del GABA, que es un neurotransmisor implicado en procesos de inhibición de la actividad neuronal, y del glutamato, que tiene una función excitatoria y regula los estados de alerta a través de sus conexiones talámicas ${ }^{26}$.

En resumen, la hipótesis más sostenible para explicar la fisiopatología del delirium plantea alteraciones de la neurotransmisión que implicarían una hipofunción colinérgica, con aumento simultáneo de la liberación de dopamina y de serotonina. Así, ante la presencia de agresiones, la reserva cerebral de cada paciente determinaría la posibilidad de que la neurotransmisión que regula diversas funciones de integración ubicadas fundamentalmente en el hemisferio derecho, se organizase de manera adaptativa, o fracasara y diera lugar al cuadro clínico de delirium.

\section{CONSIDERACIONES CLÍNICAS}

Los criterios operativos que permiten el diagnóstico de delirium más utilizados son los que propone la APA (Asociación Americana de Psiquiatría) que aparecen en la Tabla 4. Sin embargo, conviene aclarar que desde el punto de vista clínico, existen varios tipos de delirium: el hipoactivo (con bajo nivel de conciencia y disminución de la actividad psicomotora), el hiperactivo (con aumento de la alerta e inquietud o agitación psicomotora) y el mixto, combinación de fluctuaciones entre los dos subtipos anteriores ${ }^{9}$. Liptzin y Levkoff ${ }^{31}$ estudiaron la frecuencia de los distintos subtipos de delirium en 325 pacientes admitidos en un hospital general y encontraron 15\% de hiperactivos, $19 \%$ de hipoactivos, $52 \%$ de tipo mixto, y $14 \%$ que no se clasificó: no hubo diferencias significativas en cuanto a edad, sexo, lugar de residencia o diagnóstico concomitante de demencia. 


\section{Tabla 4. D SM -IV-T R: C riterios de delirium}

A. Alteración de conciencia con reducción de la capacidad de focalizar, mantener o cambiar la atención.

B. Cambios en las funciones cognoscitivas (como déficit de memoria, desorientación, alteraciones de lenguaje) o presencia de una alteración perceptiva que no se explica por la existencia de una demencia previa o en desarrollo.

C. La alteración se presenta en un corto período de tiempo (habitualmente en horas o días) y tiende a fluctuar a lo largo del día.

D. Demostración a través de la historia, de la exploración física y de las pruebas de laboratorio de que la alteración es un efecto fisiológico directo de una enfermedad médica, por intoxicación 0 abstinencia de sustancias o debido a múltiples etiologías.

La afectación generalizada de las funciones superiores se traduce, en última instancia, en deterioro en la capacidad de pensar y evaluar correctamente el entorno ${ }^{8,32}$, al que el paciente responderá con alteraciones de la conducta. Estas alteraciones pueden implicar riesgos para él y para otros, ya que incluyen actitudes hostiles y conductas de auto y heteroagresividad. Al mismo tiempo, estas manifestaciones clínicas aparatosas pueden confundir a los profesionales y propiciar errores diagnósticos y terapéuticos. Por ejemplo, puede suceder que pacientes en estas circunstancias sean dados de alta a petición propia, con riesgo para su salud, a pesar de que no están en condiciones de tomar decisiones y cuidar de sí mismos.

Por otro lado, por la naturaleza fluctuante del cuadro, la sintomatología se presentará en forma oscilante durante el día con tendencia a empeorar por la noche y con una profunda alteración del ciclo sueño-vigilia, razón por la que se hace imprescindible la valoración de un cuidador cercano (ya sea familiar del paciente o parte del equipo sanitario) para poder realizar una adecuada historia de la sintomatología del paciente. Esta misma característica debe obligar al equipo médico a ser cuidadoso con las solicitudes o exigencias del paciente que se debe considerar que no está en condiciones de tomar decisiones, lo que deberá considerarse en la evaluación médico-legal de algunas de sus conductas. Asimismo, en relación con la inclusión del paciente en protocolos de investigación, deberá contarse con el consentimiento informado de un familiar o tutor responsable.

\section{DiAgNóstico}

El diagnóstico del delirium sigue siendo básicamente clínico, aunque el estudio electroencefalográfico permitirá observar un patrón de ondas lentas, expresivo del daño metabólico cerebral ${ }^{26}$, que es notablemente sensible pero poco específico. En las formas moderadas de delirium puede haber disminución en la frecuencia de ritmos dominantes posteriores, sin ondas alfa, y en las formas más graves puede observarse una disminución de frecuencia de ondas tetha y delta. El EEG tiende a normalizarse a medida que disminuye la sintomatología del delirium, y en el caso de que su aparición esté relacionada con el consumo de alcohol se observa actividad rápida de bajo voltaje y enlentecimiento durante la intoxicación etílica ${ }^{26}$.

Desde hace unos años se han prodigado los instrumentos de evaluación clínica basados en criterios diagnósticos que intentan aumentar la sensibilidad y la especificidad en la detección del síndrome. Buena parte de los instrumentos utilizados para detectar el delirium de hecho se han diseñado para detectar demencia. El más utilizado en nuestro medio es el Mini Examen Cognoscitivo33 (versión española del Mini-mental State Examination ${ }^{34}$ ), que ofrece información de interés para detectar deterioro cognoscitivo, pero que no permite diferenciar entre delirium y demencia. De los instrumentos restantes, dos son los que actualmente tienen mayor difusión, a partir de sus validaciones de origen:

a) Delirium Rating Scale ${ }^{35,36}$ (DRS), que es útil para valorar la gravedad del delirium pero no 
para detectarlo. Es un instrumento de aplicación compleja para clínicos no especialistas y requiere bastante tiempo para su administración.

b) Confusion Assessment Method 21 (CAM), que está pensado para facilitar el diagnóstico de delirium en el hospital general a profesionales no especialistas en psiquiatría. Es un instrumento diseñado a partir de los criterios operativos del DSM III R y precisa de pocos minutos para su administración. Proporciona un algoritmo para establecer el diagnóstico en torno a la presencia de sus dos criterios fundamentales (inicio abrupto con fluctuación de síntomas y pérdida de la atención) y de uno de los dos criterios secundarios (pensamiento desorganizado y alteración del nivel de conciencia). En la actualidad, nuestro grupo de trabajo está adaptando y validando este instrumento diagnóstico en población española ${ }^{37}$.

En la Tabla 5 se expone el diagnóstico diferencial del delirium con otros síndromes psiquiátricos, como la demencia, los estados depresivos y la esquizofrenia, de los que se distingue básicamente por su aparición abrupta y su curso fluctuante.

\section{PREVENCIÓN}

La prevención con protocolos estandarizados, da aplicación en los pacientes más susceptibles, constituye la medida más eficaz para disminuir la aparición del delirium. Inouye et $\mathrm{al}^{38}$, en un estudio randomizado y controlado, demostraron que el abordaje en las áreas que se preven de más riesgo, podría reducir el número y la duración de los episodios de delirium en pacientes hospitalizados de edad avanzada. La intervención consiste en actuar sobre los seis factores de riesgo de delirium: déficit cognoscitivo, abstinencia de fármacos o sustancias, inmovilidad, déficit visual, déficit auditivo, y deshidratación. El delirium se presentó en 9,9\% de los pacientes intervenidos y en $15 \%$ del grupo no sometido a intervención, siendo los factores más importantes el adecuado control hidroelectrolítico, el uso correcto de fármacos y el tratamiento de los síntomas de privación sensorial, a partir de medidas ambientales. Estas medidas incluyen la repetición de fecha y lugar donde se encuentra el paciente, orientación horaria, calendario visible, explicaciones accesibles, estimulación por parte de los familiares, iluminación y temperatura adecuadas, evitación de ruidos para facilitar el descanso nocturno, uso de correctores sensoriales (como gafas 0 audífonos) y de objetos personales, y movilización adecuada (por lo menos, tres veces al día, en períodos no inferiores a $15 \mathrm{~min})^{8,9,22,38}$.

En los pacientes agitados es importante llevar a cabo una contención física adecuada, revisable periódicamente, al tiempo que se destaca al personal y a la familia que se trata de una medida de protección para evitar posibles lesiones, caídas, dificultades en el manejo de enfermería (sondas, flebotomías, cuidado de heridas perioperatorias) e

Tabla 5. D iagnóstico diferencial

\begin{tabular}{|lllll|}
\hline & Demencia & Depresión & Esquizofrenia & Delirium \\
\hline Inicio & Insidioso & Subagudo & Subagudo & Agudo \\
Curso $24 \mathrm{~h}$ & Estable & Estable & Estable & Fluctuante \\
Conciencia & Vigil & Vigil & Vigil & Alterado \\
Orientación & Alterada & Intacta & Intacta & Alterada \\
Memoria & Deterioro & Conservada & Conservada & Deterioro \\
Atención & Déficit parcial & Déficit parcial & Déficit parcial & Déficit grave \\
Delirios y Alucinaciones & Baja frecuencia & Baja frecuencia & Frecuentes & Frecuentes \\
\hline
\end{tabular}


incluso la posibilidad de que el paciente se autolesione o lesione a terceros. El paciente agitado tendrá también otros requerimientos hidroelectrolíticos cuyo control habrá que establecer en cada caso. Como ya se mencionó anteriormente, estos pacientes pueden pasar de estados de agitación a estados de inhibición, y de estados de confusión total a estados de considerable claridad de conciencia, y es en estos momentos cuando habrá que explicar al paciente su situación y los motivos de las medidas de contención.

\section{TRATAMIENTO}

El tratamiento ha de ser etiológico y estar basado en el estudio de los posibles factores causales, y en la identificación y neutralización de los factores desencadenantes ${ }^{9}$. En segundo término se deberá instaurar tratamiento sintomático con medicamentos o medidas ambientales, con el fin de controlar los síntomas conductuales o psicóticos y mejorar las funciones cognoscitivas ${ }^{5-7}$.

El tratamiento con neurolépticos es el tratamiento de elección para los síntomas psicóticos del delirium, por tanto son útiles especialmente en los delirium hiperactivos (delirios, alucinaciones, agitación psicomotora) aunque también estarían indicados en los delirium hipoactivos, en los que actuarían mejorando la integración de las funciones cognitivas ${ }^{8}$. Además, los neurolépticos tienen una efectividad terapéutica rápida y son superiores a las benzodiacepinas en los casos de delirium que no son debidos a privación de alcohol o de tranquilizantes ${ }^{9}$.

El haloperidol sigue considerándose por muchos autores como el neuroléptico de elección, por su equilibrio entre la efectividad antipsicótica y la escasez de efectos colaterales (pocos efectos adversos circulatorios, anticolinérgicos y cardiotóxicos, no depresión del centro respiratorio y posible administración por cualquier vía, incluyendo la intravenosa) $)^{1-9}$. Las dosis variarán según la vía de administración, la edad del paciente y la presencia de efectos adversos extrapiramidales. Se sugiere el uso inicial de una dosis creciente de 1$2 \mathrm{mg} /$ día, que se puede incrementar en intervalos de una hora hasta $10 \mathrm{mg} /$ día según la respuesta obtenida. Si no se puede controlar la agitación, o es muy aparatosa, se sugiere el uso de $2,5 \mathrm{mg}$ im o iv cada hora, hasta controlar los síntomas o hasta que aparezcan efectos secundarios extrapiramidales.

Con la aparición de los nuevos neurolépticos, se ha logrado reducir de forma sustancial los efectos adversos de los neurolépticos clásicos, con una eficacia similar en cuanto a la remisión de los síntomas psicóticos ${ }^{9,39-42}$, gracias a que estos fármacos bloquean más selectivamente la neurotransmisión dopaminérgica y serotonérgica: la olanzapina a dosis de 2,5 a $10 \mathrm{mg}$ y la risperidona a dosis entre 1 y $6 \mathrm{mg}$ han demostrado su seguridad y efectividad 39,41 , y un perfil equilibrado en el tratamiento de los síntomas psicóticos en pacientes geriátricos ${ }^{43,44}$.

En el tratamiento de los deliria causados por abstinencia de alcohol o tranquilizantes, es fundamental instaurar un tratamiento sustitutivo con benzodiacepinas, que jugarán el doble papel de prevenir el delirium y sedar al paciente. Su asociación con neurolépticos estará indicada para hacer posible un equilibrio que permita el tratamiento etiológico y sintomático. Dentro de las benzodiacepinas se sugiere el uso de loracepam, por su fácil metabolización, su vida media corta, y su improbable acumulación, y habrá que ajustar las dosis según la edad, el peso, la función hepática, la magnitud de los síntomas de abstinencia y el consumo previo ${ }^{9}$.

Recientemente, se han abierto líneas de investigación con fármacos procolinérgicos (inhibidores de la acetilcolinesterasa) ${ }^{8}$ y con mediadores en la respuesta de las citocinas ${ }^{45}$, pero no hay datos concluyentes que avalen su eficacia clínica.

\section{CONCLUSIONES}

El delirium es un trastorno de alta incidencia en los hospitales generales, donde a menudo se infradiagnóstica, y en nuestra experiencia, recibe menor atención de la debida por parte de los clínicos. Es un síndrome que genera alta mortalidad y morbilidad en los pacientes que lo sufren, y que genera elevados costes sanitarios, como consecuencia directa de la prolongación de las estancias hospitalarias y de las complicaciones médicas a que da lugar. El delirium es especialmente frecuente en pacientes hospitalizados de edad avanzada, que reciben tratamientos complejos 0 
que presentan más de una patología, de manera que es aconsejable una conducta de constante alerta por parte de todo el equipo sanitario para su detección precoz y su correcto tratamiento. Puesto que las medidas de control ambiental están libres de efectos adversos y han demostrado su eficacia, se deberían instaurar en todos los hospitales mediante protocolos estandarizados dirigidos

\section{REFERENCIAS}

1. Lipowski ZJ. Delirium: Acute Confusional States. New York, Oxford University Press, 1990.

2. Folstein MF, Bassett SS, Romanoski AJ, Nestadt G. The epidemiology of delirium in the community: the eastern Baltimore mental survey, in International Psychogeriatrics. Edited by Miller NE, Lipowski ZJ, Lebowitz BD. New York, Springer, 1991; 169-76.

3. Lipowski ZJ. Update of Delirium. Psychiatr Clini North Am 1992; 15: 335-46.

4. DSM IV TR. Manual diagnóstico y estadístico de los trastornos mentales. Ed Barcelona Madrid Masson cop. 2002.

5. CIE-10. Trastornos mentales y del comportamiento: Criterios diagnósticos de investigación. Organización Mundial de la Salud. 1993.

6. INOUYE SK. The dilemma of delirium: clinical and research controversies regarding diagnosis and evaluation of delirium in hospitalized elderly medical patients. Am J Med 1994; 97: 278-88.

7. TRZPACS PT. Update on the neuropathogenesis of delirium. Dement and geriat cogn 1999; 10: 3304.

8. MeAgher JM. Delirium: optimizing management. BMJ 2001; 322: 144-50.

9. Textbook of Consultation-Liaison Psychiatry, 2a ed. Washington DC. The American Psychiatric Publishing 2002.

10. FIEMINGER S. Remembering delirium. BMJ 2002; 180: $4-5$.

11. Lipowski ZJ. Delirium in the elderly patient. N Engl J Med 1989; 320: 578-82. al control de los factores predisponentes y desencadenantes que pueden provocar el cuadro clínico, especialmente en las poblaciones más susceptibles. Esta labor preventiva también debería ir acompañada de procedimientos de detección precoz, tratamiento y seguimiento del trastorno para controlar sus posibles consecuencias en la evolución del paciente.

12. Inouye SK, Charpentier PA. Precipitating factors for delirium in hospitalized elderly patients: predictive model and interrelationship with baseline vulnerability. JAMA 1996; 275: 852-7.

13. Navinés R, Gómez E, Franco JG, de Pablo J. Delirium en la interconsulta psiquiátrica de un hospital general. Actas Esp de Psiquiatr 2001; 29: 159-69.

14. Valdés M, de Pablo J, Campos R, Farré JM, Girón M, LozANo $\mathrm{M}$ ET AL. El proyecto multinacional europeo y multicéntrico español de mejora de calidad asistencial en psiquiatría de enlace en el hospital general: el perfil clínico en España. Med Clin (Barc) 2000; 155: 690-4.

15. Inouye SK. Delirium in hospitalized older patients: Recognition and risk factors. J of Geriat Psych Neur 1998; 11: 118-25.

16. Fann JR, Roth-Roemer S, Brington BE, Katon WJ, Syrjala KL. Delirium in patients undergoing hematopoietic stem cell transplantation. Cancer 2002; 95: 1971-81.

17. GonzÁlez J, Barros J. Delirium in patients of an intermediate care unit: prospective study. Rev Méd Chile 2000; 128: 75-9.

18. Ltaker D, Locala J, Franco K, Bronson DL, Tannous Z. Preoperative risk factors for postoperative delirium. Gen Hosp Psychiatry 2001; 23: 84-9.

19. Marcantonio ER, Fiacker JM, Wright RJ, Resnick NM. Reducing delirium after hip fracture: a randomized trial. J Am Geriatr Soc 2001; 49: 51622.

20. UlDAL KK, Berghuis JP. Delirium in AIDS patients: recognition and medication factors. AIDS Patient Care STDS 1997; 11: 435-41. 
21. Inouye SK, VAn Dyck CH, Alessi CA, Balkin S, SiegAL AP, Horwitz RL. Clarifying Confusion: The Confusion Assessment Method. A new method for detection of delirium. Ann Intern Med 1990; 113: 941-8.

22. Moss JH, Lanctot KL. Iatrogenic Benzodiazepine withdrawal Delirium in hospitalized older patients. JAGS 1995; 43: 1020-2.

23. McCusker J, Cole M, Dendukuri N, Belzile E, Primeau F. Delirium in older medical inpatients and subsequent cognitive and functional status: a prospective study. CMAJ 2001; 165: 575-83.

24. Inouye SK, Rushing JT, Foreman MD, Palmer RM, Poмpei P. Does delirium contribute to poor hospital outcomes? A three-site epidemiologic study. J Gen Intern Med 1998; 13: 234-42.

25. Rizzo JA, Bogardous ST Jr, Leo-Summers L, Wiwams CS, ACAMPORA D, INOUYe SK. Multicomponent targeted intervention to prevent delirium in hospitalized older patients: what is the economic value? Med Care 2001; 39: 740-52.

26. TRZEPACZ PT. The neuropathogenesis of delirium. Psychosomatics 1994; 35: 374-91.

27. Tune LE, Dainloth NF, Holand A. Association of postoperative delirium with raised serum levels of anticholinergics drugs. Lancet 1981; 2: 651-3.

28. COFFMan JA, Disalver SC. Cholinergic mechanisms in delirium. Am J Psychiatry 1988; 145: 382-3.

29. Mussi C, FerRari R, Ascari S, Salviol G. Importance of serum anticholinergic activity in the assessment of elderly patients with delirium. J Geriatr Psychiatry Neurol 1999; 12: 82-6.

30. MuLer WE, Stol L, Schubert T et al. Central cholinergic functioning and aging. Acta Psychiatr Scand 1991; 366: 34-9.

31. LipTZin B, LevKoff SE. An empirical study of delirium subtypes. Br J Psychiatry 1992; 161: 8435.

32. Brown TM, Boyle MF. Delirium, Clinical Review. BMJ 2002; 325: 644-7.

33. Lobo A, Saz P, Marcos G, Día JL, de la Camara C, VENTURA T. Revalidation and standardization of the mini-exam (first Spanish version of the MiniMental Status Examination) in the general geriatric population. Med Clin (Barcelona) 1999; 112: 767-74.
34. Folstein MF, Folstein SE, McHugh PR. "Minimental state": a practical method for grading the cognitive state of patients for the clinician. J Psychiatr Res 1975; 12: 189-98.

35. TRzepacs PT, Baker RW, Greenhouse J. A symptom rating scale for delirium. Psychiatry Res 1988; 23: 89-97.

36. Bulbena A, Corrons C, Amorós G, Martín-Santos R, Anguiano B. Escalas de delirium y orientación. Adaptación castellana del Delirium Rating Scale y de la Orientation Scale. Rev Gerontología 1996; 6: 245-54.

37. González M, Fuente E, Nomdedeu M, Peri JM, de PABLO J, VALDÉs M. Adaptación de un instrumento de detección de delirium. Actas Esp Psiquiatri 2002; Vol. 18, 33.

38. Inouye SK, Bogardus ST, Charpentier PA, LeoSummers L, ACAMpora D, Holford TR et al. A multicomponet intervention to prevent delirium in hospitalized older patients. N Eng J Med 1999; 340: 669-76.

39. SchwarTZ TL, Masand PS. The role of atypical antipsychotics in the treatment of delirium. Psychosomatics 2002; 43: 171-4.

40. Chan YC, Pariser SF, Neufeld G. Atypical antipsychotics in older adults. Pharmacotherapy 1999; 19: 811-22.

41. Breitbart W, Trembiay A, Gibson C. An open trial of olanzapine for the treatment of delirium in hospitalized cancer patients. Psychosomatics 2002; 43: 175-82.

42. Sipahimalani A, Masand PS. Olanzapine in the treatment of delirium. Psychosomatics 1998; 39: 422-30.

43. Street JS, Ciark WS, Gannon KS, Cummings JL, BYMATER FP, TAMURA RN ET AL. Olanzapine treatment of psychotic and behavioral symptoms Alzheimer disease in nursing care facilities: a double-blind placebo controlled trial. The HGEU study group. Arch Gen Psychiatry 2000; 57: 968-76.

44. Street JS, Tolefeson GD, Tohen M, Sanger TM, Clark WS, Gannon KS et aL. Olanzapine for psychotic conditions in the elderly. Psychiatric Annals 2000; 30: 191-6.

45. BRoadhurst C, WiLSON K. Immunology of delirium: new opportunities for treatment and research. BJP 2001; 179: 288-9. 